\title{
Prevention and Treatment of Acute Respiratory Distress Syndrome by COVID-19: Role of Vitamin D on Immunomodulation and Renin-Angiotensin- Aldosterone System Regulation
}

\author{
Maximiliano Carmona ${ }^{1}$, Virna M Martín Giménez ${ }^{2}$ and Walter Manucha ${ }^{3,4 *}$ \\ ${ }^{1}$ Hospital Luis Carlos Lagomaggiore, Ministerio de Salud, Desarrollo Social y Deportes, Gobierno de Mendoza, Argentina \\ ${ }^{2}$ Instituto de Investigaciones en Ciencias Químicas, Facultad de Ciencias Químicas y Tecnológicas, Universidad Católica de Cuyo, \\ Sede San Juan, Argentina
}

${ }^{3}$ Laboratorio de Farmacología Experimental Básica y Traslacional. Área de Farmacología, Departamento de Patología, Facultad de Ciencias Médicas, Universidad Nacional de Cuyo, Mendoza, Argentina

${ }^{4}$ Instituto de Medicina y Biología Experimental de Cuyo, Consejo Nacional de Investigación Científica y Tecnológica (IMBECU-CONICET), Mendoza, Argentina

*Corresponding author: Walter Manucha, Farmacología, Depto Patología Facultad de Ciencias Médicas UN de Cuyo Centro Universitario, Mendoza, Argentina

\section{ARTICLE INFO}

Received: 㓞 October 12, 2021

Published: 幽 October 21, 2021

Citation: Maximiliano Carmona, Virna M Martín Giménez, Walter Manucha. Prevention and Treatment of Acute Respiratory Distress Syndrome by COVID-19: Role of Vitamin D on Immunomodulation and Renin-Angiotensin-Aldosterone System Regulation. Biomed J Sci \& Tech Res 39(3)-2021. BJSTR. MS.ID.006314.

Keywords: Vitamin D; 1,25(OH)2D; Calcitriol; COVID-19; SARS-CoV-2; Acute Respiratory Distress Syndrome

\begin{abstract}
The leading cause of complications or death from COVID-19 is due to the development of acute respiratory distress syndrome (ARDS). This syndrome is characterized by exacerbated inflammation, mainly at the pulmonary level, and an over-activation of the renin-angiotensin-aldosterone system (RAAS), usually culminating in death or permanent respiratory sequelae in the SARS-CoV-2 infected patients. In this sense, it has been proposed that vitamin $\mathrm{D}$ exerts numerous immunomodulatory effects by acting on various types of immune and epithelial cells, as well as being able to stimulate the protective arm (ACE2/Ang-(1-7)) and inhibit the harmful arm (ACE/Ang-II) of the RAAS. Therefore, some of the main mechanisms underlying these effects mediated by vitamin $\mathrm{D}$ and its receptors are discussed in the present review. The analysis of these mechanisms would positively influence the prevention and treatment of ARDS, thus preventing the progression of the disease and improving the prognosis of the patients.

Abbreviations: ARDS: Acute Respiratory Distress Syndrome; ICU: Intensive Care Unit; RAAS: Renin-Angiotensin-Aldosterone System; VD: Vitamin D; VDR: Vitamin D Receptors; LPS: Lipopolysaccharides; ACE: Angiotensin Converting Enzyme; ERK: Extracellular Signal-Regulated Kinases
\end{abstract}

\section{Introduction}

The development and severity of the acute respiratory distress syndrome (ARDS) observed in patients with coronavirus disease 2019 (COVID-19) is a potentially fatal condition and, at the same time, a determining factor in the prognosis of it pathology [1]. In this sense, a study developed by Quesada-Gómez and colleagues reported that approximately $20 \%$ of hospitalized patients [both in the intensive care unit (ICU) and not admitted to the ICU] with COVID-19 developed ARDS and, despite recent improvements in the strategies of mechanical ventilation and supportive care, around $65 \%$ of patients with ARDS died [2]. ARDS is a fundamental component of the pathophysiology of this disease, whereby patients 
with severe COVID-19 develop multi-organ dysfunction. The onset of ARDS is usually rapid and progressive, developing approximately nine days after the onset of severe COVID-19 [3] infection. The disease has been challenging to manage in terms of high morbidity and mortality, high costs and long periods of hospitalization in intensive care. Therefore, early treatment is essential to control the progression of the infection and improve the prognosis of patients with ARDS [4,5]. ARDS develops as a consequence of an exaggerated or uncontrolled inflammatory response known as a "cytokine storm", resulting from the release of large concentrations of these proteins (IFN- $\alpha$, IFN- $\gamma$, IL-1 $\beta$, IL-6, IL-12, IL-18, IL-33, TNF- $\alpha$, TGF- $\beta$, CCL2, CCL3, CCL5, CXCL8, CXCL9, CXCL10, etc.) proinflammatory by immune effector cells during SARS-CoV-2 [6] infection. Furthermore, the activation and excessive recruitment of neutrophils into the alveolar interstitium with disruption of the endothelial-epithelial barrier and the consequent alveolar damage exacerbates the pathogenesis of ARDS [7]. Of particular interest, the data obtained to date from patients infected with SARS-CoV-2 confirmed that those who required admission to the ICU had higher concentrations of GCSF, IP10, MCP1, MIP1A, and TNF- $\alpha$ than those that did not require it, which reaffirms the fact that the intensity of the cytokine storm is usually associated with the severity of the disease [8].

Currently, multiple therapeutic behaviors are used -some empirically based and others based on limited evidence- with varying efficiencies for ARDS management, mainly associated with supportive care such as mechanical ventilation and hemodynamic management [9]. Of particular interest, it is known that vitamin D (VD) and its receptors (VDR) are present in all living beings that are part of the animal kingdom, playing a fundamental role in the maintenance of bone health and phospho-calcium metabolism. However, the fact that VD precursors have also been found in invertebrate organisms such as krill and phytoplankton [10] highlights their importance in regulating numerous additional physiological and homeostatic processes, such as controlling inflammation and oxidative stress [11,12]. Therefore, VD could play a leading role in the prevention and treatment of oxidative and inflammatory diseases such as COVID-19 by attenuating several of the underlying mechanisms involved in the pathophysiology of this viral infection. VD deficiency is associated with an increased risk of respiratory infection [13-16]. Likewise, a recent metaanalysis involving around 10,000 individuals from 25 health centers concluded that VD supplementation reduced the risk of upper respiratory tract infections by approximately 19\%, with patients being severely deficient in VD who experienced the greatest benefit [17]. Additionally, VD has demonstrated, in in vitro models, its ability to inhibit the production and release of cytokines (IFN- $\alpha$, IFN- $\gamma$, IL-1 $\beta$, IL-6, IL-12, IL-18, IL-33, and TNF- $\alpha$ ) and chemokines (CCL2, CCL3, CCL5, CXCL8, CXCL9, and CXCL10) involved in the development and progression of ARDS [18]. Indeed, it has been reported that VD and VDR signaling may provide some beneficial effects in ARDS through multiple mechanisms, including attenuation of the cytokine and chemokine storm [19], regulation of the renin-angiotensin-aldosterone system (RAAS) [20] and the maintenance of the integrity of the pulmonary epithelial barrier [21], among other aspects, which will be addressed in this review.

\section{Role of Vitamin D in Immuno-Modulation and its Potential Efficacy during COVID-19}

More than a century ago (1849), the British physician C.J.B. Williams reported that in a study of 234 tuberculosis patients, 206 of them showed a "marked and unequivocal improvement" after treatment with cod liver oil (rich in VD) [22]. Since then, various VD functions related to the regulation of numerous cellular processes have been investigated, concluding that its deficiency is potentially involved in the development of multiple diseases [23]. Additionally, since the discovery of the presence of VDR in a wide variety of cells of the immune system, countless studies on the immunomodulatory effect of RV, that is, its ability to stimulate (e.g. to accelerate the elimination of a pathogen) or inhibit (e.g. to attenuate uncontrolled inflammation) the immune response according to specific circumstances. In detail, these studies revealed that VD would modulate the activity of various immune cells, including monocytes, macrophages, lymphocytes and epithelial cells [24]. In the case of macrophages, it has been observed that low serum concentrations of VD in patients with rickets correlate with a decrease in their phagocytic activity, which could be reversed by supplementation with 1,25-dihydroxyvitamin D3 (1,25-(OH )2D3), or also called calcitriol, the active form of VD [25]. For its part, it has been reported that the antimicrobial activity of macrophages increases in the presence of 25-hydroxyvitamin D3 (25-(OH)D3) after stimulation with antigen ligands.

This is because the activation of different macrophage receptors (TLR-2, TLR-4, among others) by foreign agents leads to an increased expression of VDR and of the CYP27B enzyme, which increases both the conversion of 25-(OH)D3 into its active form 1,25- $(\mathrm{OH}) 2 \mathrm{D} 3$ mediated by the CYP27B enzyme, as well as from the expression of antimicrobial peptides such as cathelicidin and $\beta 2$-defensin through VDR $[26,27]$. Both peptides contribute to hosting defenses by stimulating the expression of antiviral cytokines and chemokines involved in the recruitment of monocytes/macrophages, neutrophils and T lymphocytes, among other immune cells [28]. Another mechanism by which VD can promote innate immune function through antimicrobial peptides is closely linked to improving phagocytosis through the induction of autophagy [29]. SARS-CoV-2 uses blocking autophagy to accelerate 
its replication and infectivity. By achieving this, the virus induces the expression of the Skp2 protein, which plays a central role in the viral replication mechanism during COVID-19 disease and, in turn, inactivates Beclin 1, a component of the autophagic process [30,31]. In parallel, 1,25-(OH)2D3 also stimulates the production of Klotho, known to attenuate multi-organ aging and increase longevity, which also promotes autophagy through the maintenance of adequate cellular levels of Beclin 1 [32]. Concerning B lymphocytes, $1,25-(\mathrm{OH}) 2 \mathrm{D} 3$ has been shown to play an important role in the homeostasis of these cells through the inhibition of proliferation and the induction of apoptosis in activated B cells. Likewise, 1,25- $(\mathrm{OH})$ 2D3 inhibits the differentiation of B lymphocytes to plasma cells and memory B cells. These mechanisms can prevent or attenuate the pathogenesis of diseases involving B lymphocytes [33]. About $\mathrm{T}$ lymphocytes, the biologically active $1,25-(\mathrm{OH}) 2 \mathrm{D} 3$ is capable of modifying the immune response, shifting it from a T helper 1 (proinflammatory) profile to a T helper 2 (anti-inflammatory) profile. Additionally, 1,25-(OH)2D3 directly affects the differentiation of regulatory $\mathrm{T}$ cells and modulates the functions of dendritic cells [34]. As for the respiratory epithelium cells, they express enzymes capable of converting the precursor 25-(OH)D3 into its active form 1,25-(OH)2D3.

Therefore, these cells constitute an important source of 1,25- $(\mathrm{OH}) 2 \mathrm{D} 3$ that induces the expression of cathelicidin and CD14 by cells of the immune system [35]. Furthermore, type II cuboidal alveolar cells of the lung also express VDR to a large extent. Thus, VDR overexpression exerts a pulmonary antiinflammatory effect $[36,37]$. VDR knockout mice experienced more severe acute lung injury than wild-type mice after being treated with lipopolysaccharides (LPS) [38]. Calcitriol/VDR signaling can also protect against acute lung injury by modulating genes that regulate the expression of occluding, a protein responsible for the maintenance of alveolar epithelial tight junctions, avoiding the increased permeability of the epithelial-alveolar barrier stimulated by the inflammatory answer. Therefore, $1,25(\mathrm{OH}) 2 \mathrm{D}$ is essential to maintain the structure and function of the epithelial barriers of the lung, among other tissues [21]. In this sense, the mechanisms described could represent potential immunomodulatory pathways against the SARS-CoV-2 virus induced by VD, outlined in Figure 1.

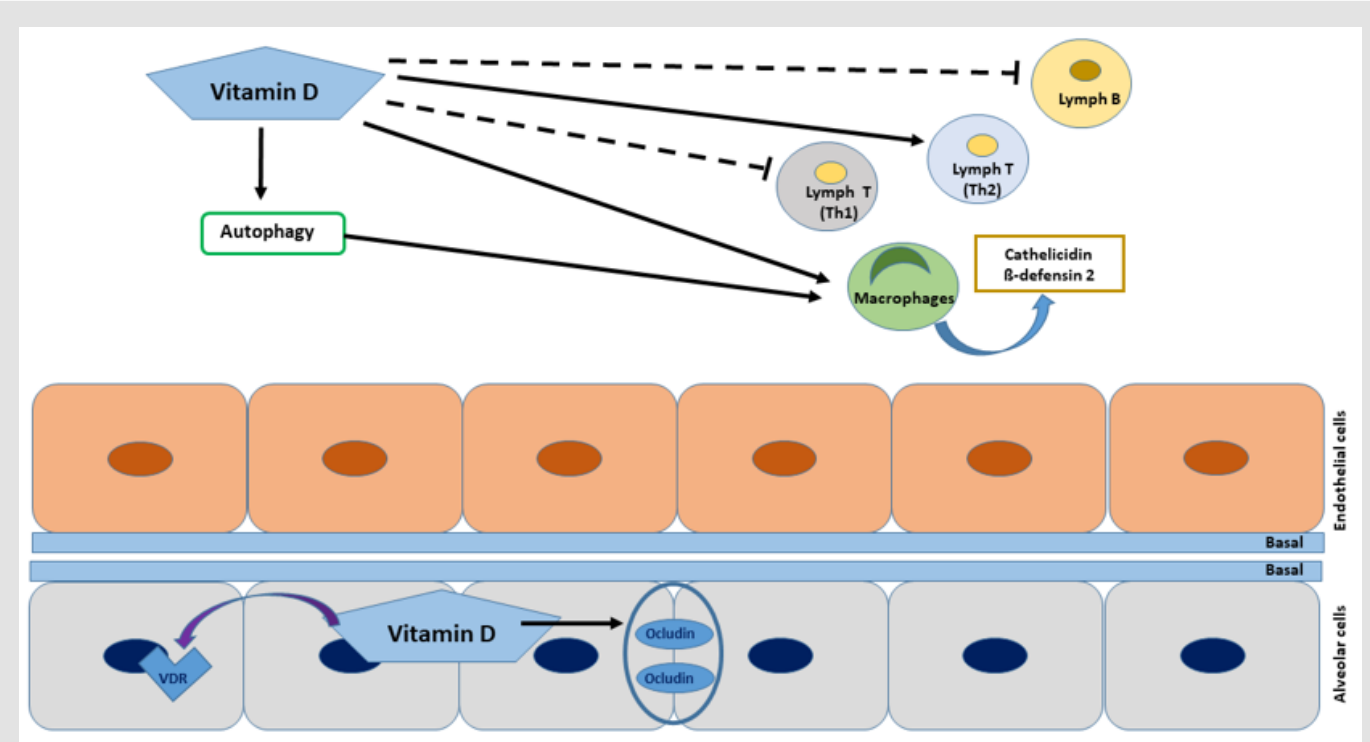

Figure 1: Potential immuno-modulating mechanisms of vitamin D at the endothelial-alveolar barrier level against infection by the SARS-CoV-2 virus. Solid arrows indicate "stimulation", and broken arrows indicate "inhibition."

\section{Role of the Renin Angiotensin System in the Pathogenesis of COVID-19 and its Relationship with the Protective Role of Vitamin D}

Local or systemic inflammatory responses can activate RAAS. Likewise, the hyper-activation of RAAS induced by SARS-CoV-2 together with the consequent decrease in angiotensin-converting enzyme 2 (ACE2), have been implicated in the pathogenesis of ARDS by COVID-19 [39]. Thus, angiotensin II (Ang-II) generated by angiotensin converting enzyme 1 (ACE) is capable of inducing lung injury, while ACE2 counter-regulates the effects produced by Ang-II by converting Ang II into Ang-(1-7), which in turn activates the Mas receptor to repress the STAT3 signaling pathways and extracellular signal-regulated kinases (ERK), acting as an anti-inflammatory factor. Therefore, the alteration of the balance between the levels of the enzymes ACE and ACE2, affect the endogenous relationship of Ang-II/Ang-(1-7), with elevation of Ang-II, this being a key factor for the development of ARDS in animal and human models [40]. Additionally, it is important to note that SARS-CoV-2 does not enter cells through coronavirus receptors such as aminopeptidase 
$\mathrm{N}$ and dipeptidyl peptidase 4, but rather through the ACE239, [40] receptor. In this sense, approximately $83 \%$ of the cells that express the ACE2 receptor are a type II alveolar epithelial cell, which suggests that these cells can serve as a reservoir for viral invasion, further complicating the situation at the pulmonary level [41]. Interestingly, it has been observed that the expression of ACE2 (but not that of ACE) is significantly reduced in lung tissue during SARS-CoV-2 [42] infection. In this way, a crucial lung protection mechanism is lost, and the SARS-CoV-2 infection becomes more lethal, with recruitment, accumulation and exaggerated activation of neutrophils and macrophages, increased vascular permeability due to the breakdown of the barrier epithelial-alveolar (with diffuse alveolar damage and exacerbated edema at the pulmonary level), which will eventually lead to ARDS. In this state, the lungs cannot provide enough oxygen to the blood, which is essential for the proper functioning of vital organs [43]. Additionally, this state of hyper-inflammation and the over-activation of the RAAS are intimately involved in the alteration of the coagulation cascade, which leads to a prothrombotic state, commonly observed in ARDS during SARS-CoV-2 infection $[44,45]$. Another consequence of COVID-19 is the potential risk of fibrosis with impaired lung function in recovered patients $[46,47]$.

In this sense, there is ample evidence that 1,25(OH)2D/VDR signaling is a potent negative regulator of RAAS [13,19,20]. Renin levels are elevated in VDR knock-out mice. Chronic VD deficiency can induce activation of RAAS. 1,25(OH)2D can reduce renin, ACE and Ang-II levels, simultaneously increasing ACE2 levels during lipopolysaccharide-induced acutelung injury [48].Xu and colleagues conducted a study on rat lung endothelial cells to determine whether calcitriol at concentrations of 5, 20, and 100nM affects lipopolysaccharide-induced acute lung injury. The results indicated that the higher concentration of calcitriol (100nM) significantly reduced the deleterious effects of lipopolysaccharides on ACE and ACE2 levels, suggesting an essential role for the active form of VD in attenuating acute lung injury. In parallel, and considering that the type 1 Ang-II receptor (AT1) is a downstream effector of ACE, the effects of lipopolysaccharides and calcitriol on its expression were then investigated. In line with the results described above, a significant increase in AT1 mRNA expression was observed in cells exposed to lipopolysaccharides compared to those that were not, while calcitriol reduced the effect of lipopolysaccharides on AT1 mRNA levels in a dose-dependent manner. It should be noted that the mRNA expression of the Ang-II type 2 receptor (AT2), used as a control, was not significantly altered. Therefore, these results confirm that calcitriol can prevent lipopolysaccharide-induced acute lung injury in rat lung endothelial cells, at least partly due to ACE2/Ang-(1-7) axis activation and inhibition of the ACE/Ang-II/ AT1R axis of the RAS [49].

On the other hand, we must consider that 1,25(OH)2D also positively regulates ACE2; thus VD could facilitate the uptake of viruses in cells that express this enzyme in their membranes. This dual effect means that ACE2 can potentially behave both in a harmful and beneficial way, which generated controversies and intense debates related to using Ang-II antagonists in hypertensive patients [50]. Despite this, most experts concluded that the beneficial effects of ACE2 on the lungs would negate its role in the entry of the virus into these cells [51]. Figure 2 outlines the relationship between VD and RAAS in the context of COVID-19.

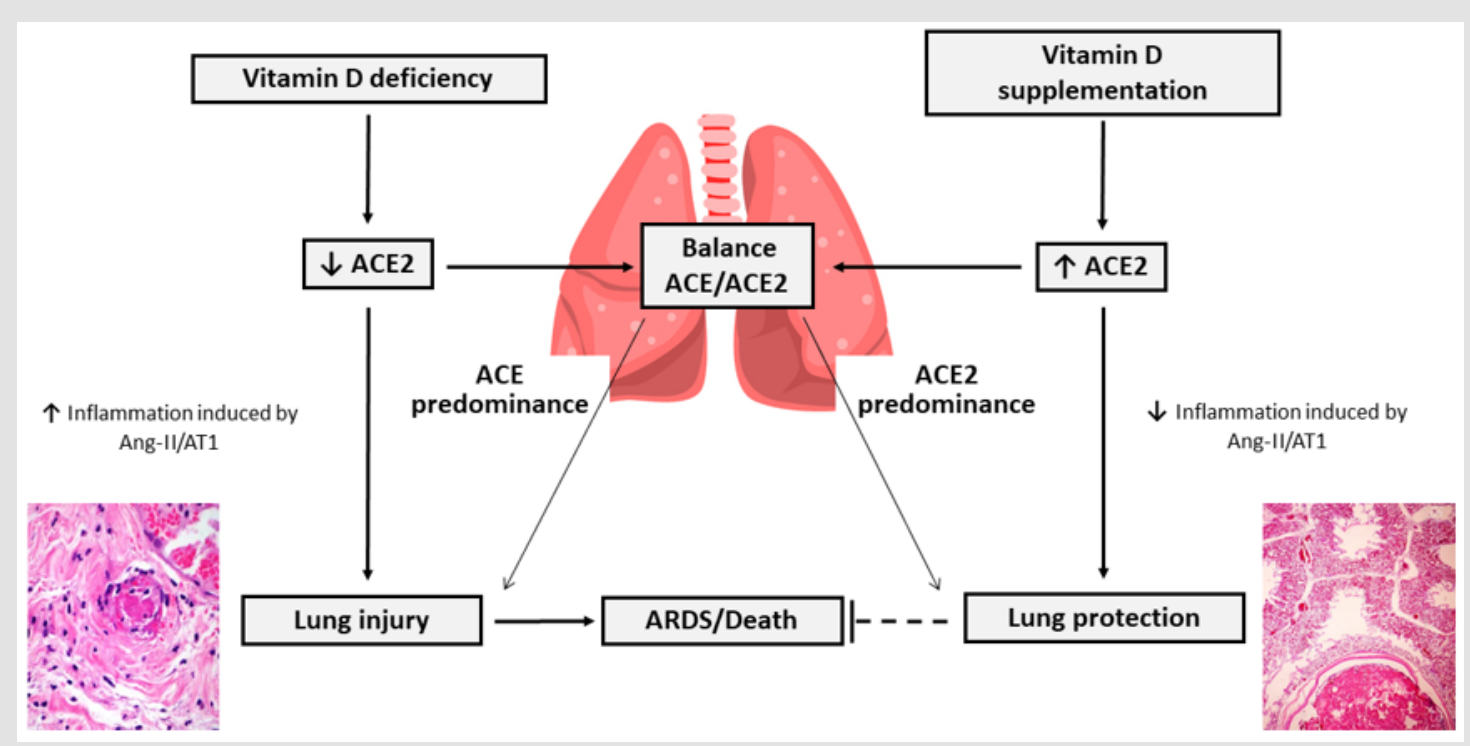

Figure 2: Regulation of the renin-angiotensin-aldosterone system by vitamin D and its protective effects during COVID-19. Solid arrows indicate "stimulation", and broken arrows indicate "inhibition." 


\section{Conclusion and Perspectives}

Numerous studies indicate that VD deficiency is associated with increased morbidity and mortality in patients with COVID-19. Furthermore, it has been suggested that VD supplementation would reduce the risk of developing ARDS in these individuals. However, the underlying molecular mechanisms of VD in the prevention and attenuation of this respiratory condition during COVID-19 are still not fully understood, which is crucial to develop better therapeutic management that allows saving the lives of patients infected with SARS- CoV-2. Currently, several clinical trials are being carried out worldwide to evaluate the effects of VD supplementation in patients with COVID-19, both individually and in combination with other active ingredients, comparing the effect of high doses versus standard doses. However, the available evidence does not allow VD supplementation as an authorized therapy in patients admitted to health care centers. Therefore, as a perspective, we propose the challenge of generating new evidence through multicenter clinical studies based on VD supplementation to patients with COVID-19 and applying the knowledge of critical analysis in order to obtain successful results.

\section{Authors' Contribution}

All authors contributed in the same way in the conception and design of the review, with a substantial contribution on the data, analysis and interpretation of the contents, writing and critical review of the article for its intellectual content.

\section{Declaration of Conflict of Interest}

The authors declare no potential conflicts of interest concerning the research, authorship and/or publication of this article.

\section{Financial Support}

The authors declare that they have not received financial support for the research, authorship and/or publication of this article.

\section{References}

1. Batah SS, Fabro AT (2021) Pulmonary pathology of ARDS in COVID-19: A pathological review for clinicians. Respir Med 176: 106239

2. Quesada Gomez JM, Entrenas Castillo M, Bouillon R (2020) Vitamin D receptor stimulation to reduce acute respiratory distress syndrome (ARDS) in patients with coronavirus SARS-CoV-2 infections: Revised Ms SBMB 2020_166. J Steroid Biochem Mol Biol 202(105719): 105719.

3. Li X, Ma X (2020) Acute respiratory failure in COVID-19: is it "typical" ARDS? Crit Care 24(1): 198.

4. Ferguson ND, Pham T, Gong MN (2020) How severe COVID-19 infection is changing ARDS management. Intensive Care Med 46(12): 2184-2186.

5. Fan E, Beitler JR, Brochard L, Calfee CS, Ferguson ND, et al. (2020) COVID-19-associated acute respiratory distress syndrome: is a different approach to management warranted? Lancet Respir Med 8(8): 816-821.
6. Lin SH, Zhao YS, Zhou DX, Zhou FC, Xu F (2020) Coronavirus disease 2019 (COVID-19): cytokine storms, hyper-inflammatory phenotypes, and acute respiratory distress syndrome. Genes Dis 7(4): 520-527.

7. Yang SC, Tsai YF, Pan YL, Hwang TL (2020) Understanding the role of neutrophils in acute respiratory distress syndrome. Biomed J 20: S23194170 .

8. Kox M, Waalders NJB, Kooistra EJ, Gerretsen J, Pickkers P (2020) Cytokine Levels in Critically Ill Patients With COVID-19 and Other Conditions. JAMA 324(15): 1565-1567.

9. Peck TJ, Hibbert KA (2019) Recent advances in the understanding and management of ARDS. F1000Res 8: F1000.

10. Hernigou P, Auregan JC, Dubory A (2018) Vitamin D: part I; from plankton and calcified skeletons (500 million years ago) to rickets. Int Orthop 42(9): 2273-2285.

11. Ferder M, Inserra F, Manucha W, Ferder L (2013) The world pandemic of vitamin D deficiency could possibly be explained by cellular inflammatory response activity induced by the renin-angiotensin system. Am J Physiol Cell Physiol 304(11): C1027-1039.

12. De Las Heras N, Martín Giménez VM, Ferder L, Manucha W, Lahera $\mathrm{V}$ (2020) Implications of Oxidative Stress and Potential Role of Mitochondrial Dysfunction in COVID-19: Therapeutic Effects of Vitamin D. Antioxidants (Basel) 9(9): 897.

13. Ferder L, Martín Giménez VM, Inserra F, Tajer C, Antonietti L, et al. (2020) Vitamin D supplementation as a rational pharmacological approach in the COVID-19 pandemic. Am J Physiol Lung Cell Mol Physiol 319(6): L941-L948.

14. Martín Giménez VM, Inserra F, Ferder L, García J, Manucha W (2021) Vitamin D deficiency in African Americans is associated with a high risk of severe disease and mortality by SARS-CoV-2. J Hum Hypertens 35(4): 378-380.

15. Mariani J, Giménez VMM, Bergam I, Tajer C, Antonietti L, et al. (2021) Association Between Vitamin D Deficiency and COVID-19 Incidence, Complications, and Mortality in 46 Countries: An Ecological Study. Health Secur 19(3): 302-308.

16. Martín Giménez VM, Ferder L, Inserra F, García J, Manucha W (2020) Differences in RAAS/vitamin D linked to genetics and socioeconomic factors could explain the higher mortality rate in African Americans with COVID-19. Ther Adv Cardiovasc Dis 14: 1753944720977715.

17. Martineau AR, Jolliffe DA, Greenberg L, Aloia JF, Bergman P, et al. (2019) Vitamin D supplementation to prevent acute respiratory infections: individual participant data meta-analysis. Health Technol Assess 23(2): $1-44$.

18. Bui L, Zhu Z, Hawkins S, Cortez Resendiz A, Bellon A (2021) Vitamin D regulation of the immune system and its implications for COVID-19: A mini review. SAGE Open Med 9: 20503121211014073.

19. Martín Giménez VM, Inserra F, Tajer CD, Mariani J, Ferder L, et al. (2020) Lungs as target of COVID-19 infection: Protective common molecular mechanisms of vitamin D and melatonin as a new potential synergistic treatment. Life Sci 254: 117808.

20. Giménez VMM, Sanz RL, Marón FJM, Ferder L, Manucha W (2020) Vitamin D-RAAS Connection: An Integrative Standpoint into Cardiovascular and Neuroinflammatory Disorders. Curr Protein Pept Sci 21(10): 948-954

21. Chen H, Lu R, Zhang YG, Sun J (2018) Vitamin D Receptor Deletion Leads to the Destruction of Tight and Adherens Junctions in Lungs. Tissue Barriers 6(4): 1-13.

22. Karbalaei M, Ghazvini K, Keikha M (2020) Clinical efficacy of vitamin D supplementation on pulmonary TB patients: The evidence of clinical trials. J Clin Tuberc Other Mycobact Dis 20: 100174. 
23. Wang H, Chen W, Li D, Yin X, Zhang X, et al. (2017) Vitamin D and Chronic Diseases. Aging Dis 8(3): 346-353.

24. Charoenngam N, Holick MF (2020) Immunologic Effects of Vitamin D on Human Health and Disease. Nutrients 12(7): 2097.

25. Djukic M, Onken ML, Schütze S, Redlich S, Götz A, et al. (2014) Vitamin $\mathrm{d}$ deficiency reduces the immune response, phagocytosis rate, and intracellular killing rate of microglial cells. Infect Immun 82(6): 25852594.

26. Chung C, Silwal P, Kim I, Modlin RL, Jo EK (2020) Vitamin D-Cathelicidin Axis: at the Crossroads between Protective Immunity and Pathological Inflammation during Infection. Immune Netw 20(2): e12.

27. Youssef DA, Miller CW, El Abbassi AM, Cutchins DC, Cutchins C, et al. (2011) Antimicrobial implications of vitamin D. Dermatoendocrinol 3(4): 220-229.

28. Ahmed A, Siman Tov G, Hall G, Bhalla N, Narayanan A (2019) Human Antimicrobial Peptides as Therapeutics for Viral Infections. Viruses 11(8): 704

29. Germic N, Frangez Z, Yousefi S, Simon HU (2019) Regulation of the innate immune system by autophagy: neutrophils, eosinophils, mast cells, NK cells. Cell Death Differ 26(4): 703-714.

30. Shojaei S, Suresh M, Klionsky DJ, Labouta HI, Ghavami S (2020) Autophagy and SARS-CoV-2 infection: Apossible smart targeting of the autophagy pathway. Virulence 11(1): 805-810.

31. Gassen NC, Niemeyer D, Muth D, Corman VM, Martinelli S, et al. (2019) SKP2 attenuates autophagy through Beclin1-ubiquitination and its inhibition reduces MERS-Coronavirus infection. Nat Commun 10(1): 5770 .

32. Berridge MJ (2017) Vitamin D deficiency accelerates ageing and agerelated diseases: a novel hypothesis. J Physiol 595(22): 6825-6836.

33. Rolf L, Muris AH, Hupperts R, Damoiseaux J (2014) Vitamin D effects on B cell function in autoimmunity. Ann N Y Acad Sci 1317: 84-91.

34. Keleş E, Özkara Ş, ilhan N, Güngör H, Karlıdağ T, et al. (2015) The Relationship between Th1/Th2 Balance and 1 $\alpha$, 25-Dihydroxyvitamin D3 in Patients with Allergic Rhinitis. Turk Arch Otorhinolaryngol 53(4): 139-143.

35. Schrumpf JA, Van der Does AM, Hiemstra PS (2020) Impact of the Local Inflammatory Environment on Mucosal Vitamin D Metabolism and Signaling in Chronic Inflammatory Lung Diseases. Front Immunol 11: 1433.

36. Tsujino I, Ushikoshi Nakayama R, Yamazaki T, Matsumoto N, Saito I (2019) Pulmonary activation of vitamin D3 and preventive effect against interstitial pneumonia. J Clin Biochem Nutr 65(3): 245-251.

37. Hu G, Dong T, Wang S, Jing H, Chen J (2019) Vitamin D3-vitamin D receptor axis suppresses pulmonary emphysema by maintaining alveolar macrophage homeostasis and function. EBio Medicine 45: 563577.
38. Shi YY, Liu TJ, Fu JH, Xu W, Wu LL, et al. (2016) Vitamin D/VDR signaling attenuates lipopolysaccharide induced acute lung injury by maintaining the integrity of the pulmonary epithelial barrier. Mol Med Rep 13(2): 1186-1194.

39. Zemlin AE, Wiese OJ (2020) Coronavirus disease 2019 (COVID-19) and the renin-angiotensin system: A closer look at angiotensin-converting enzyme 2 (ACE2). Ann Clin Biochem 57(5): 339-350.

40. Wiese OJ, Allwood BW, Zemlin AE (2020) COVID-19 and the reninangiotensin system (RAS): A spark that sets the forest alight? Med Hypotheses 144: 110231.

41. Li G, He X, Zhang L, Ran Q, Wang J, et al. (2020) Assessing ACE2 expression patterns in lung tissues in the pathogenesis of COVID-19. J Autoimmun 112: 102463.

42. Verdecchia P, Cavallini C, Spanevello A, Angeli F (2020) The pivotal link between ACE2 deficiency and SARS-CoV-2 infection. Eur J Intern Med 76: 14-20.

43. Zhang X, Li S, Niu S (2020) ACE2 and COVID-19 and the resulting ARDS. Postgrad Med J 96(1137): 403-407.

44. Abou Ismail MY, Diamond A, Kapoor S, Arafah Y, Nayak L (2020) The hypercoagulable state in COVID-19: Incidence, pathophysiology, and management. Thromb Res 194: 101-115.

45. Becker RC (2020) COVID-19 update: Covid-19-associated coagulopathy. J Thromb Thrombolysis 50(1): 54-67.

46. Rai DK, Kumar S, Sahay N (2021) Post-COVID-19 pulmonary fibrosis: A case series and review of literature. J Family Med Prim Care 10(5): 20282031.

47. Rai DK, Sharma P, Kumar R (2021) Post covid 19 pulmonary fibrosis. Is it real threat? Indian J Tuberc 68(3): 330-333.

48. Dancer RC, Parekh D, Lax S, D’Souza V, Zheng S, et al. (2015) Vitamin D deficiency contributes directly to the acute respiratory distress syndrome (ARDS). Thorax 70(7): 617-624.

49. Xu J, Yang J, Chen J, Luo Q, Zhang Q et al. (2017) Vitamin D alleviates lipopolysaccharide induced acute lung injury via regulation of the renin angiotensin system. Mol Med Rep 16(5): 7432-7438.

50. Lo KB, McCullough PA, Rangaswami J (2020) Antihypertensive drugs and risk of COVID-19? Lancet Respir Med 8(5): e29.

51. Semenzato L, Botton J, Drouin J, Baricault B, Vabre C, et al. (2021) Antihypertensive Drugs and COVID-19 Risk: A Cohort Study of 2 million Hypertensive Patients. Hypertension 77(3): 833-842. 
ISSN: 2574-1241

DOI: $10.26717 /$ BJSTR.2021.39.006314

Walter Manucha. Biomed J Sci \& Tech Res

(c) (P) This work is licensed under Creative BY Commons Attribution 4.0 License

Submission Link: https://biomedres.us/submit-manuscript.php

\begin{tabular}{ll} 
BIOMEDICAL & \multicolumn{1}{c}{ Assets of Publishing with us } \\
RESEARCHES & - Global archiving of articles \\
\hline Immediate, unrestricted online access
\end{tabular}

